\title{
Manufacturing and evaluation of multi-channel cylinder applicator with 3D printing technology
}

\author{
Reza Mohammadi, MScl, Zahra Siavashpour, PhD², Seyed Rashid Hosseini Aghdam, PhD³, Samar Fazli, MSc ${ }^{4}$ \\ Tibor Major, PhD 5,6, Ali Asghar Rohani, MSc ${ }^{7}$ \\ 'Department of Medical Physics, Iran University of Medical Sciences, Tehran, Iran, ${ }^{2}$ Department of Radiotherapy Oncology, Shohada-e \\ Tajish Educational Hospital, Shahid Beheshti University of Medical Sciences, Tehran, Iran, ${ }^{3}$ Department of Medical Radiation Engineering. \\ Shahid Beheshti University, Tehran, Iran, ${ }^{4}$ IRA Radiotherapy Center, National Center of Oncology V.A. Fanarjian Hospital, Yerevan, Armenia. \\ ${ }^{5}$ Department of Oncology, Semmelweis University, Budapest, Hungary, ${ }^{6}$ Center of Radiotherapy, National Institute of Oncology, Budapest, \\ Hungary. ${ }^{7}$ Department of Medical Physics, Tehran University of Medical Sciences, Tehran, Iran
}

\begin{abstract}
Purpose: This study was designed to assess dosimetric characteristics of 3D-printed personalized multi-channel cylinder applicator (MCCA).

Material and methods: UnionTech RS Pro 600 (UnionTech, Inc., Shanghai, China) 3D printer was used for manufacturing MCCA. The geometry of MCCA was designed with Fusion 360 v.2.0.5827 (Autodesk, Inc.) software. The designed file was exported to Meshmixer v.3.5 (Autodesk, Inc.) to create three-dimensional model in stereolithography (STL) file format, which is the common file format for inputting data to 3D printers. We used high-temp resin, FLHTAM02 model (Formlabs Inc., MA, USA), as material in 3D printing process. This resin model has good resistance to high temperature and compatibility with various solvents. We created a simple cubic shape phantom for dosimetric evaluation of the applicator with Gafchromic EBT3 films. Also, Monte Carlo method was applied to simulate MCCA in the same configuration as in experimental test.

Results: The mean \pm standard deviation (SD) difference between measured and calculated doses in treatment planning system (TPS) for all control points was $0.0860 \pm 0.0393 \mathrm{~Gy}$, corresponding to $4.01 \pm 1.21 \%$. The mean \pm SD difference between doses calculated by Monte Carlo simulation and TPS for all control points was $0.0996 \pm 0.0471$ Gy, corresponding to $4.58 \pm 1.05 \%$. The mean \pm SD of dose difference between film measurement and Monte Carlo simulation for all control points was $0.0136 \pm 0.0200 \mathrm{~Gy}$, corresponding to $0.60 \pm 0.69 \%$. $P$-value for dose difference between film measurement and TPS, Monte Carlo and TPS, and film measurement and Monte Carlo were 0.7, 0.66, and 0.95, respectively.

Conclusions: Dosimetric results and mechanical accuracy of MCCA show that high-temp resin with SLA 3D printing technique can be used for producing patient-specific MCCA in brachytherapy.

J Contemp Brachytherapy 2021; 13, 1: 80-90 DOI: https://doi.org/10.5114/jcb.2021.103590

Key words: 3D printing, brachytherapy, vaginal cancer, cervical cancer.

\section{Purpose}

According to PORTEC-2 trial, including patients with endometrial cancer, women with pathological risk features, such as high-risk of relapse ( $>50$ myometrial invasion), higher grade, lymphovascular space invasion, and old age, present intermediate-risk based on post-operative radiation therapy $[1,2]$. Pelvic external beam radiotherapy (EBRT) with vaginal cuff brachytherapy and vaginal cuff brachytherapy alone are common treatments [3]. Vaginal cuff brachytherapy alone has lower gastrointestinal and genitourinary toxicities and better quality of life compared to EBRT based on patients who were randomized to pelvic EBRT or vaginal cuff brachytherapy in

PORTEC-2 trial $[4,5,6]$. Vaginal cuff brachytherapy alone is a good choice of treatment for patients who present the above-mentioned risk features [7].

Currently, there are several commercial vaginal brachytherapy applicators, which have cylindrical shape with different length, diameters, and curvature of the intrauterine probe. The diameter of applicator is limited. For instance, vaginal/rectal cylinder applicator set from Eckert \& Ziegler (BEBIG GmbH, Berlin, Germany) contains cylinders with diameter of 20, 25, and $30 \mathrm{~mm}$, and $12.5,15,35$, and $40 \mathrm{~mm}$ as optional items. In addition, variable lengths can be obtained by using cylinder segments with different lengths, but combination of different segments requires variables of lengths
\end{abstract}


due to specified length of each segment (e.g., 5, 10, 20, and $40 \mathrm{~mm}$ ).

The selection of brachytherapy technique, such as intracavitary and interstitial, is based on depth of vaginal wall invasion and distribution of the disease [8]. Single-channel cylinder applicator for lesions with depth of $5 \mathrm{~mm}$ or less is commonly used. It is difficult to deliver the dose to lesions with depth more than $5 \mathrm{~mm}$ to at least one side of vagina's wall with single-channel cylinder applicator due to symmetrical dose distribution [9]. Multi-channel cylinder applicator (MCCA), with a channel at the center with 6-7 additional channels located concentrically around the center channel, are commercially available for different loading pattern for shaping the dose distribution that may diminish bladder and rectal dose without losing the target coverage. The diameter and length of MCCA are limited, same as the single-channel cylinder applicator.

Suitable diameter for both single-channel and MCCA should be selected to improve the fitting of applicator and to achieve a gentler dose gradient. If the diameter of selected applicator does not fit the vagina wall, it might move between acquiring the planning images and treatment, which increase uncertainty of delivering the dose to patient. Also, if the diameter of selected applicator fit the vagina wall too tightly, it may cause a discomfort for patients $[10,11,12]$. The diameter of cylinder is important to correspond to the applicator inside the vagina wall. Two studies have assessed the presence of air between cylinder and vagina wall. The presence rate of air reported was in $60 \%$ and $43 \%$ of patients, leading to $27 \%$ and $16.1 \%$ of underdose of vaginal mucosa, respectively $[13,14]$.

Personalized vaginal applicators are one of the choices to overcome these limitations of commercially used vaginal applicators $[15,16,17,18,19,20,21,22]$. Traditional technique, such as mold technique, is costly and time-consuming to create personalized vaginal applicator for each patient [23]. Recently, 3D printing technology has been used in radiotherapy and brachytherapy to manufacture anthropomorphic phantoms, boluses, templates for needle guidance, mould applicators, and multi-catheter templates for interstitial brachytherapy in breast cancer patients $[24,25,26,27,28,29,30]$. The advantages of 3D printing technology compared to mold technique are its versatility, accessibility cost-effectiveness, and accuracy.

The aim of this study was to assess the dosimetric characteristics of 3D-printed personalized MCCA.

\section{Material and methods}

\section{D printer}

In this study, we used UnionTech RS Pro 600 (UnionTech, Inc., Shanghai, China) 3D printer. The volume that can be printed with this model can reach up to $600 \times 600$ $\times 500 \mathrm{~mm}^{3}$. The laser model is a solid-state frequency tripled Nd:YVO4, with a wavelength of $354.7 \mathrm{~nm}$. The nominal beam size, maximum scanning speed, and minimum layer thickness are $0.12-0.2 \mathrm{~mm}, 12 \mathrm{~m} / \mathrm{s}$, and $0.05 \mathrm{~mm}$, respectively. This printer operates based on stereolithography (SLA) technique.
SLA was the first rapid prototyping system. "In SLA process, a photopolymer is cured by a low-powder ultraviolet laser that solidifies specific areas on the surface of the liquid through a chain reaction initiated by reactive species generated by ultraviolet exposure. After the first layer of the liquid resin is cured, the platform stage is lowered slightly, allowing a new layer of liquid to cover the now-solid planar sections. Once the planar sections are completed, the prototype is then post-cured in a controlled furnace, or an ultraviolet curing apparatus, for a designated period of time, to allow final polymerization" [31,32]. The process is described in Figure 1 [33].

The resolution can be obtained by controlling the thickness of cured layer. Energy of light, which exposes the resin, specifies the thickness of cured layer. This energy can be determined by adjusting the power of light, scanning speed, and time of exposure. The resolution of traditional SLA technique has been shown to be limited by a diameter of laser beam [34]. Recently, SLA process was developed to achieve a layer thickness less than $10 \mu \mathrm{m}$, called "micro-stereolithography" [35]. Although SLA is the oldest 3D printing technique, it is the gold standard for rapid prototyping in medical application, including tissue engineering and medical devices [31].

The geometry of MCCA was designed with Fusion 360 v.2.0.5827 (Autodesk, Inc., California, USA) software as shown in Figure 2. The dimension of each designed part is summarized in Table 1 . In addition, eight channels were considered for needles. The designed file was exported to Meshmixer v.3.5 (Autodesk, Inc., California, USA) to create three-dimensional model in stereolithography (STL) file format, which is the common file format for inputting data to $3 \mathrm{D}$ printers.

We used high-temp resin, FLHTAM02 model (Formlabs Inc., MA, USA), as a material for 3D printing process. This resin model has good resistance to high temperature $\left(238^{\circ} \mathrm{C}\right)$ and compatibility with various solvents, such as isopropyl alcohol, which can be used in cleaning and disinfection processes.

After printing the MCCA, some assessments were performed. First, we evaluated the geometric accuracy

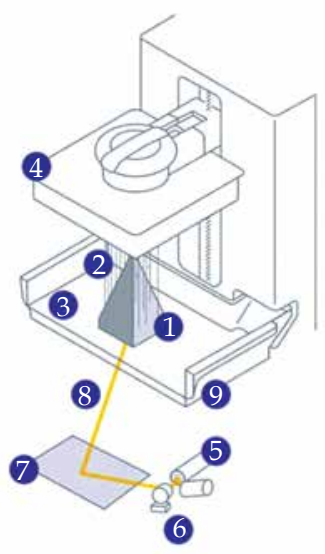

Reversed (inverted) SLA
1 Printed part
2 Supports
3 Resin
4 Build platform
5 Laser
6 Galvanometers
7 X-Y scanning mirror
8 Laser beam
9 Resin tank

Fig. 1. Diagram of stereolithography (SLA) technique process [33] 


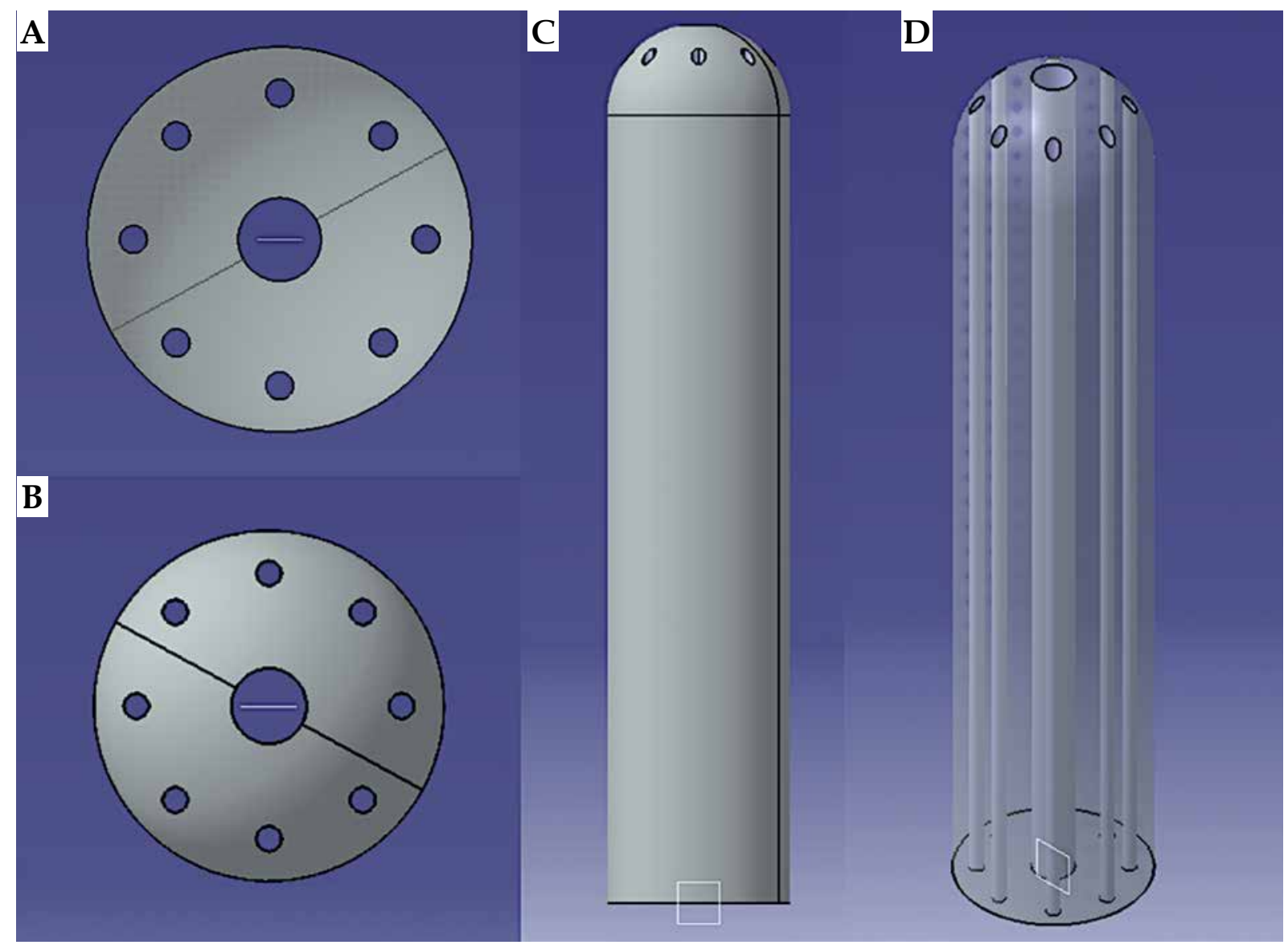

Fig. 2. The designed geometry in 4 different view. A) From bottom, B) from up, C) from lateral, D) transparent from perspective

of applicator based on physical measurement. We used absolute digital caliper model 500-150-20 (Mitutoyo Ltd., Japan), with a resolution of $0.02 \mathrm{~mm}$ to measure the dimension of applicator and compare to designed dimension. Secondly, we evaluated radiological properties of the MCCA. Computed tomography (CT) images of the 3D-printed MCCA were acquired and commercially used cylinder applicator with Somatom Spirit (Siemens Inc., Erlangen, Germany) was applied. The resolution was 0.85 $\times 0.85 \times 1 \mathrm{~mm}^{3}$ to precisely calculate Hounsfield unit (HU) and density of the applicator. The voltage and current were selected as $130 \mathrm{kV}$ and $85 \mathrm{~mA}$, respectively. Using CT images of applicator and a calibration curve in CT, the $\mathrm{HU}$ of each pixel was converted to physical density.
Then, the HU and density of MCCA were compared with a commercial cylinder.

\section{Dosimetric evaluation}

We created a simple phantom for dosimetric evaluation of the applicator with Gafchromic EBT3 films. In order to create a cubic shape phantom, we placed together 26 slabs of plexiglass with dimensions of $100 \times 100 \times$ $6 \mathrm{~mm}^{3}$ (Figure 3). Then, we used two plexiglass rods in diagonal corners of the phantom to install all of the slabs. A circular area with $25.5 \mathrm{~mm}$ as a diameter was cut by a laser in the middle of the phantom to introduce the MCCA.

The MCCA, with titanium intrauterine probe and eight steel needles, was introduced in the cubic shape

Table 1. Dimension of designed multi-channel cylinder applicator

\begin{tabular}{lccc} 
Part name & $\begin{array}{c}\text { Designed dimension } \\
(\mathrm{mm})\end{array}$ & $\begin{array}{c}\text { 3D-printed dimension } \\
(\mathrm{mm})\end{array}$ & $\begin{array}{c}\text { Differences } \\
(\mathrm{mm} \%)\end{array}$ \\
\hline Outer cylinder diameter & 25.00 & 24.90 & $0.10-0.40$ \\
\hline Intrauterine probe hole diameter & 5.80 & 5.90 & $-0.10-1.70$ \\
\hline Needles hole diameter & 1.75 & 1.80 & $-0.05-2.80$ \\
\hline Height of cylinder & 120.00 & 120.90 & $-0.90-0.75$ \\
\hline Height of needles hole & 114.00 & 114.40 & $-0.40-0.35$ \\
\hline Center of needles hole to the edge of cylinder & 3.50 & 3.55 & $-0.05-1.40$
\end{tabular}




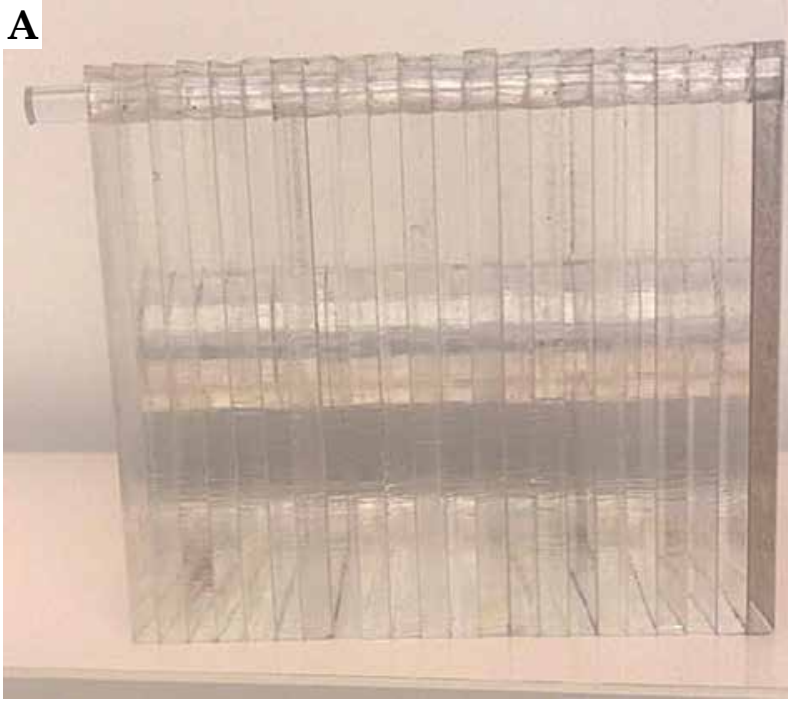

Fig. 3. Cubic shape phantom. A) Phantom from lateral view, B) phantom with MCCA (central intrauterine probe and peripheral needles)

phantom and scanned with CT scanner. The CT resolution was chosen the same as in the previous step. Then, the CT images were imported to SagiPlan v.2.0.2 brachytherapy treatment planning system (TPS, Eckert \& Ziegler BEBIG, Berlin, Germany), which uses the TG-43 dose calculation formalism with a ${ }^{60} \mathrm{Co}$ source. Four Gafchromic films in different locations between plexiglasses were placed. Planning was done based on prescription dose of $7 \mathrm{~Gy}$ at depth of $5 \mathrm{~mm}$ from the MCCA surface. $60 \mathrm{~mm}$ of both intrauterine and needles, with $16.4 \mathrm{~mm}$ and $16.8 \mathrm{~mm}$ offset from tip, respectively, were considered as activation length by dwell position separation of $1 \mathrm{~mm}$. Inverse planning was performed as a dose optimization method. Automatic control point placement option in the SagiPlan was chosen to generate points for inverse planning. This option created points at a given distance (for the cylinder radius plus $5 \mathrm{~mm}$ ) in a perpendicular to the applicator direction from selected applicator based on activated dwell positions. Then, the variance with 5 iterations was used as inverse planning algorithm. A total of 192 control points in the 4 different planes were established as shown in Figure 4. Each plane was divided by eight angles ( $45^{\circ}$ as step), and control points were set with the interval of $5 \mathrm{~mm}$ at each angle from the MCCA surface. The resolution of dose matrix was set as $1 \mathrm{~mm}$ in each direction in the TPS.

To obtain the dose-response calibration curve, we used eight samples of Gafchromic EBT3 film. They were irradiated between 100 and 900 cGy. Afterwards, a PTW Farmer-type ionization chamber model 31010 (PTW, Co., Germany) was placed in $5 \mathrm{~cm}$ depth between plexiglass layers to measure the absolute dose-rate, and $5 \mathrm{~cm}$ was chosen to establish electronic equilibrium. The source to surface distance of plexiglasses was $100 \mathrm{~cm}$ and the field size was $10 \times 10 \mathrm{~cm}^{2}$. Then, the samples were placed at the same depth $(5 \mathrm{~cm})$ and the irradiation was performed. All sam-

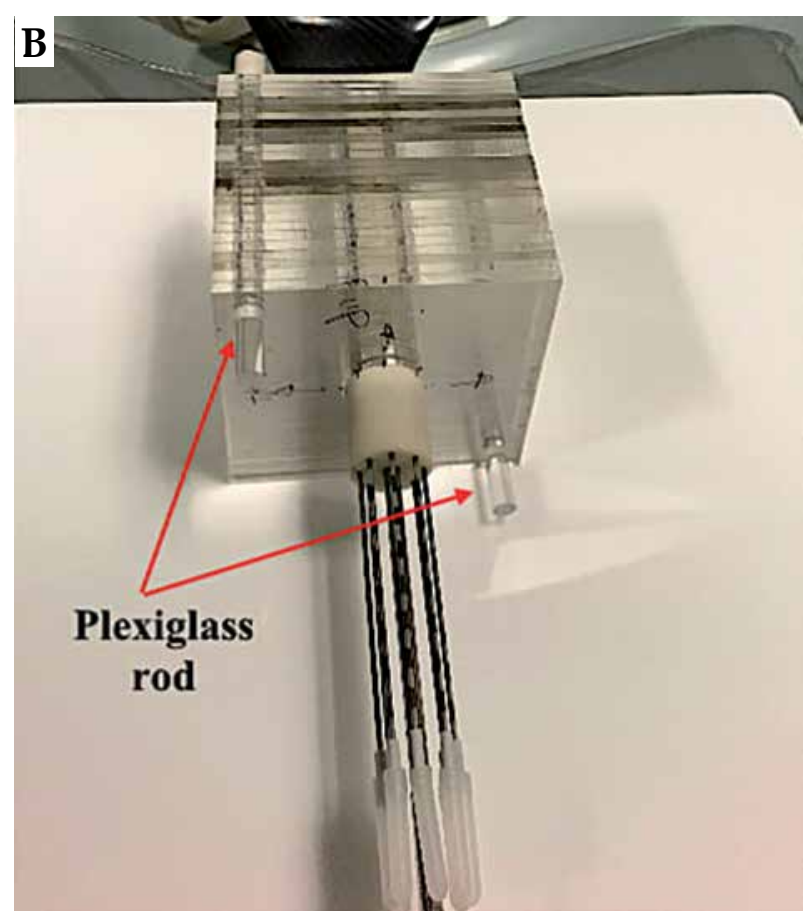

ples were handled with latex gloves and placed in darkened envelope to avoid stains and effect of ambient light.

Scanning of both measurements and calibration films were performed 48 hours after irradiation with ScanMaker 9800XL Plus (Microtek, Inc., San Diego, CA, USA). All films were scanned at the same orientation with $150 \mathrm{dpi}$ as a resolution, and saved in tagged image file format [36]. The scanned images were imported to ImageJ software v.1.52n [37]. The RGB image was split and red channel was chosen (due to maximize readout sensitivity) to calculate pixel values. The procedure of fitting curve was performed using Devic et al. [38] proposed formula. The dose of each control point from TPS was compared to measured dose from EBT3 films.

We also used a method suggested and used by Cunha et al. [19] to evaluate the resin material. We have designed a couple L-shaped to hold Gafchromic film, as shown in Figure 5A, B. The L-shaped dimension was $6 \times 3 \mathrm{~cm}^{2}$ and the distance between center of needle and Gafchromic film was $2.5 \mathrm{~mm}$. Printing was done with the same resin, which was used for the MCCA. Also, a holder designed to hold Gafchromic film for measuring in the water (Figure 5C, D) was used as validation. Printing was performed using polyamide (PA-12) material with density $=1.01 \mathrm{~g} / \mathrm{cm}^{3}$, which is water equivalent, and also placed in a water tank to provide scatter condition. Then, a $1 \mathrm{~cm}$ prescription point from the center of needle and the middle of L-shaped was applied to deliver 15 Gy. Next, Gafchromic films were scanned, and a percent reading depth between $5 \mathrm{~mm}$ and $41 \mathrm{~mm}$ from the needle were considered to compare the results.

\section{Monte Carlo simulation}

MCNP is a powerful Monte Carlo code to simulate and transport different particles and radiation in various geometries. In this study, a MCNP code was employed 

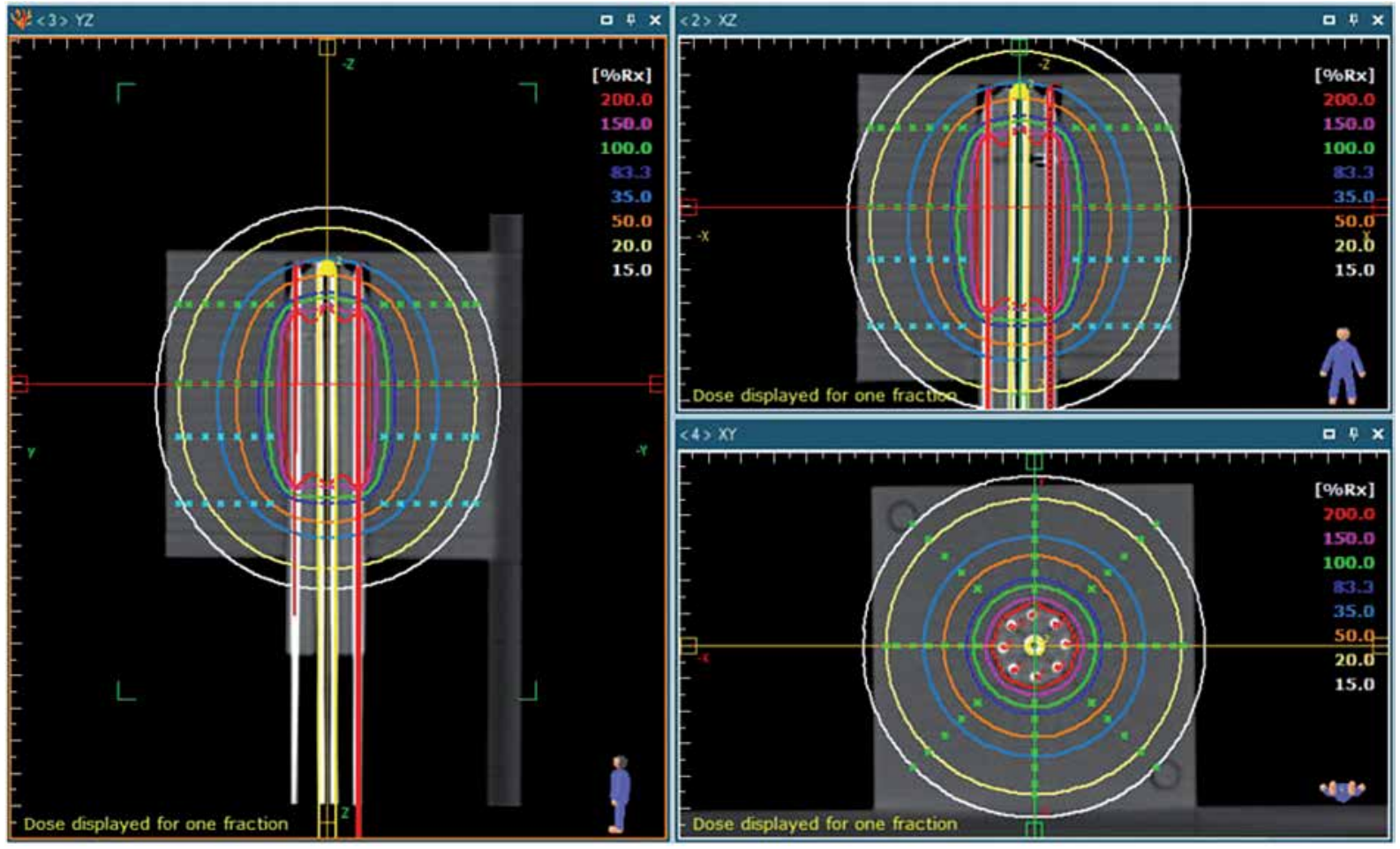

Fig. 4. Three different views of dose distribution in the phantom with control points. 192 control points set in 4 different planes. Each plane was divided by eight angles ( $45^{\circ}$ as step), and control points were set with an interval of $5 \mathrm{~mm}$ at each angle from the MCCA surface

to simulate the ${ }^{60} \mathrm{Co} \gamma$ emitter source of high-dose-rate (HDR) brachytherapy, steel needles, constructed cylinder, and the PMMA phantom in the same configuration as in the experimental test. Source activity of $65.98 \mathrm{GBq}$ ( $20.19 \mathrm{mGy} / \mathrm{h}$ as air-kerma rate) at the planning time was applied. It should be mentioned that all of the required data for simulation were taken from Eckert \& Ziegler BEBIG company.

Additionally, ${ }^{60} \mathrm{Co}$ source characteristics, including the energy intensity at two nominal energies at the surface of source housing, were considered in the simulations. The energy cut-off for electron and photon radiation was deemed to be $0.5 \mathrm{MeV}$ and $0.01 \mathrm{MeV}$, respectively. Size of scoring cells for dose calculation was set at $0.3 \times 0.25 \times$ $0.25 \mathrm{~cm}^{3}$ within the PMMA to calculate dose distribution around the applicator. In addition, the statistical uncertainty in each of the MC simulations was less than $3 \%$.

To calculate dose value from every source dwell position, nearly 500 separate MC codes were generated. *F8 tally was used to obtain energy deposition in each tally cell. The number of followed records in each MC simulation was equal to $10^{9}$. The doses equivalent distribution was shown in terms of Gy. Finally, dose distribution was summed and superimposed to achieve the total dose to each of the considered control points, and compared with TPS results.

\section{Case report}

We also designed opened tips for both intrauterine probe and needles, and compared to commercial Miami applicator set from Eckert \& Ziegler BEBIG. This helped cervix cancers patients with parametria involvement and vagina one side involvement, or great tumor width. Intrauterine probe, cylinder (printed in $30 \mathrm{~mm}$ diameter), and needles (TCN) were used simultaneously to obtain better coverage, as presented in Figure 6. The MCCA was sterilized with autoclave in $134^{\circ} \mathrm{C}$ temperature, $103 \mathrm{kPa}$ pressure, and in 35 minutes. After the insertion, two plans were generated based on 7 Gy prescription dose to high-risk clinical target volume (HR-CTV). One of them was based on using just tandem, and the other was based on using tandem plus needles.

\section{Statistical analysis}

Measured and MC calculated doses in control points were compared with independent two samples $t$-test with equal variance. Mean \pm standard deviation (SD) was applied for summarizing the data. IBM SPSS v.23 (SPSS, Inc.) was used for all statistical analyses. $P$-value less than 0.05 was considered statistically significant.

\section{Results}

The differences of geometry between the designed and 3D-printed MCCA are shown in Table 1. The mean HU and density of MCCA and commercial cylinder (polyether ether ketone, medical grade) were 252 and $1.18 \mathrm{~g} / \mathrm{cm}^{3}$, and 178 and $1.12 \mathrm{~g} / \mathrm{cm}^{3}$, respectively. The images of MCCA on CT scans were uniform, without any artifact. The percent reading depth is shown in Figure 5E, 

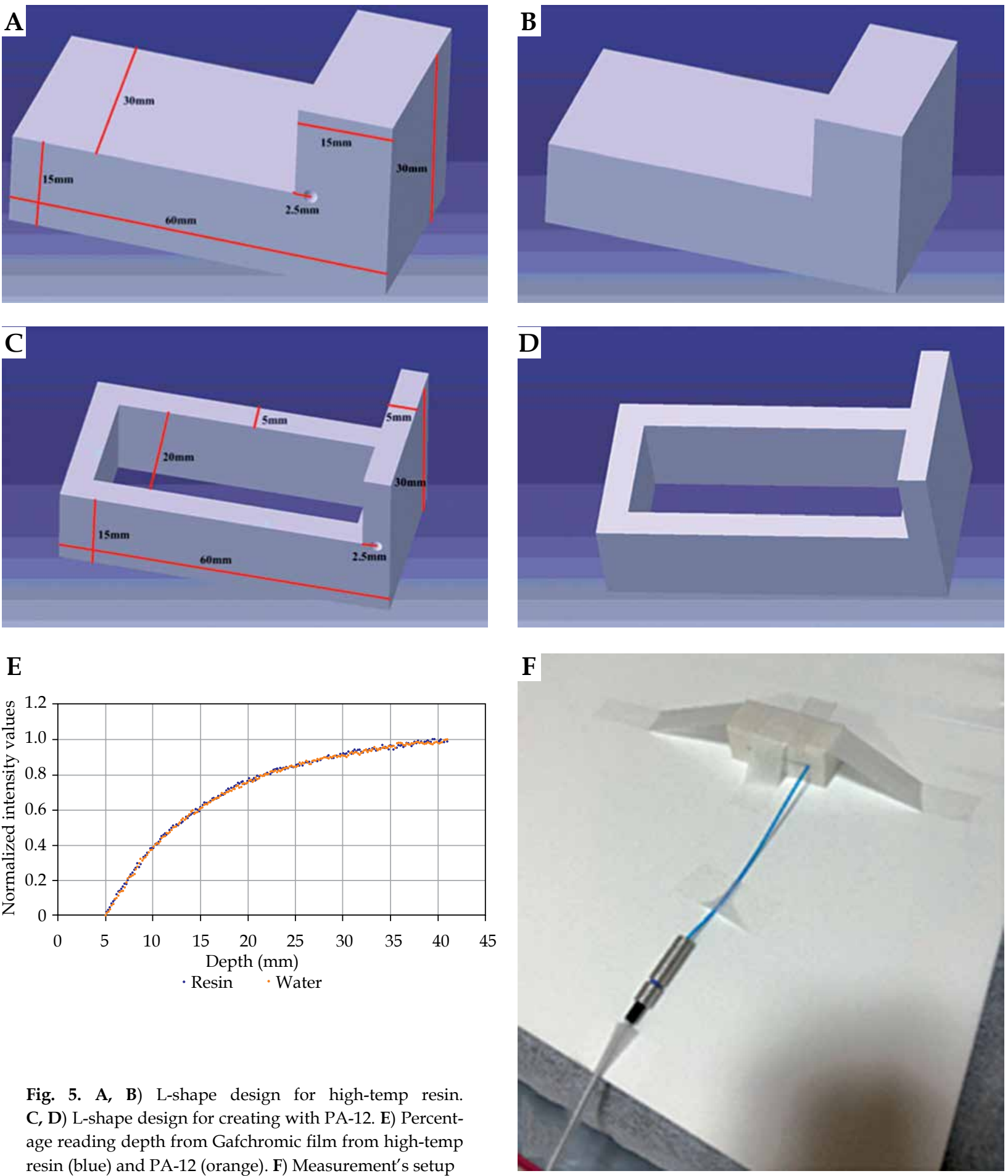

Fig. 5. A, B) L-shape design for high-temp resin. C, D) L-shape design for creating with PA-12. E) Percentage reading depth from Gafchromic film from high-temp resin (blue) and PA-12 (orange). F) Measurement's setup

which normalized to $5 \mathrm{~mm}$ from the center of needle and the differences between two curves were within $1 \%$. In addition, the mean time of 3D printing was 4 to 5 hours, and designing by an expert was $30 \mathrm{~min}$.

The mean $\pm S D$ difference between three methods are demonstrated in Table 2. The minimum and maximum difference between the measured and calculated doses in the TPS for all control points were $0.04 \mathrm{~Gy}$ and $0.22 \mathrm{~Gy}$, corresponding to $2.06 \%$ and $6.87 \%$, respectively. The minimum

and maximum difference between the doses calculated by Monte Carlo simulation and TPS for all control points were $0.05 \mathrm{~Gy}$ and $0.30 \mathrm{~Gy}$, corresponding to $2.38 \%$ and $7.72 \%$, respectively. Also, the minimum and maximum difference between film measurements and Monte Carlo simulations for all control points were $-0.04 \mathrm{~Gy}$ and $0.10 \mathrm{~Gy}$, corresponding to $-1.27 \%$ and $2.47 \%$, respectively. The measured and Monte Carlo calculated doses were less than those calculated by the planning system for all control points. Dos- 
A

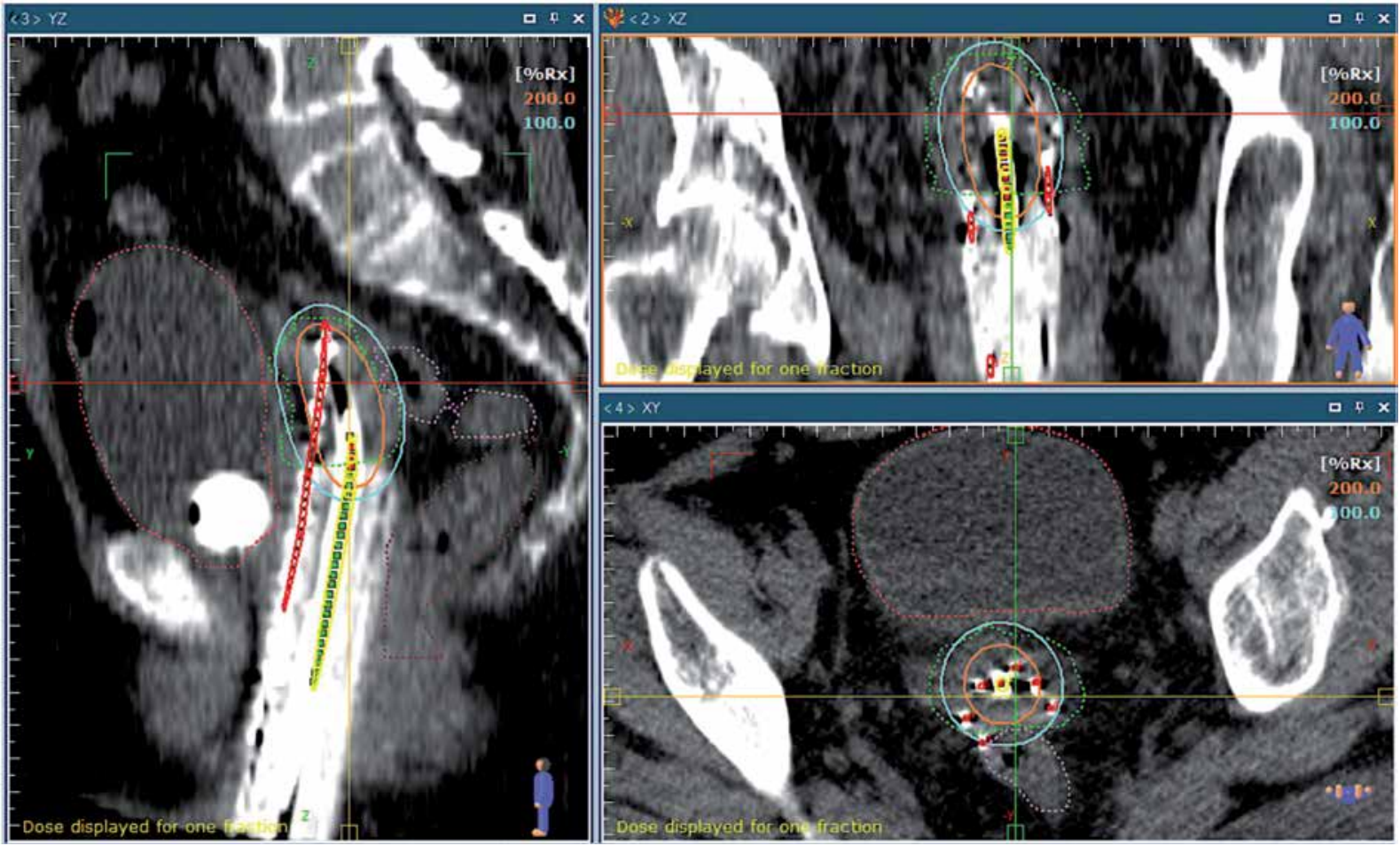

B

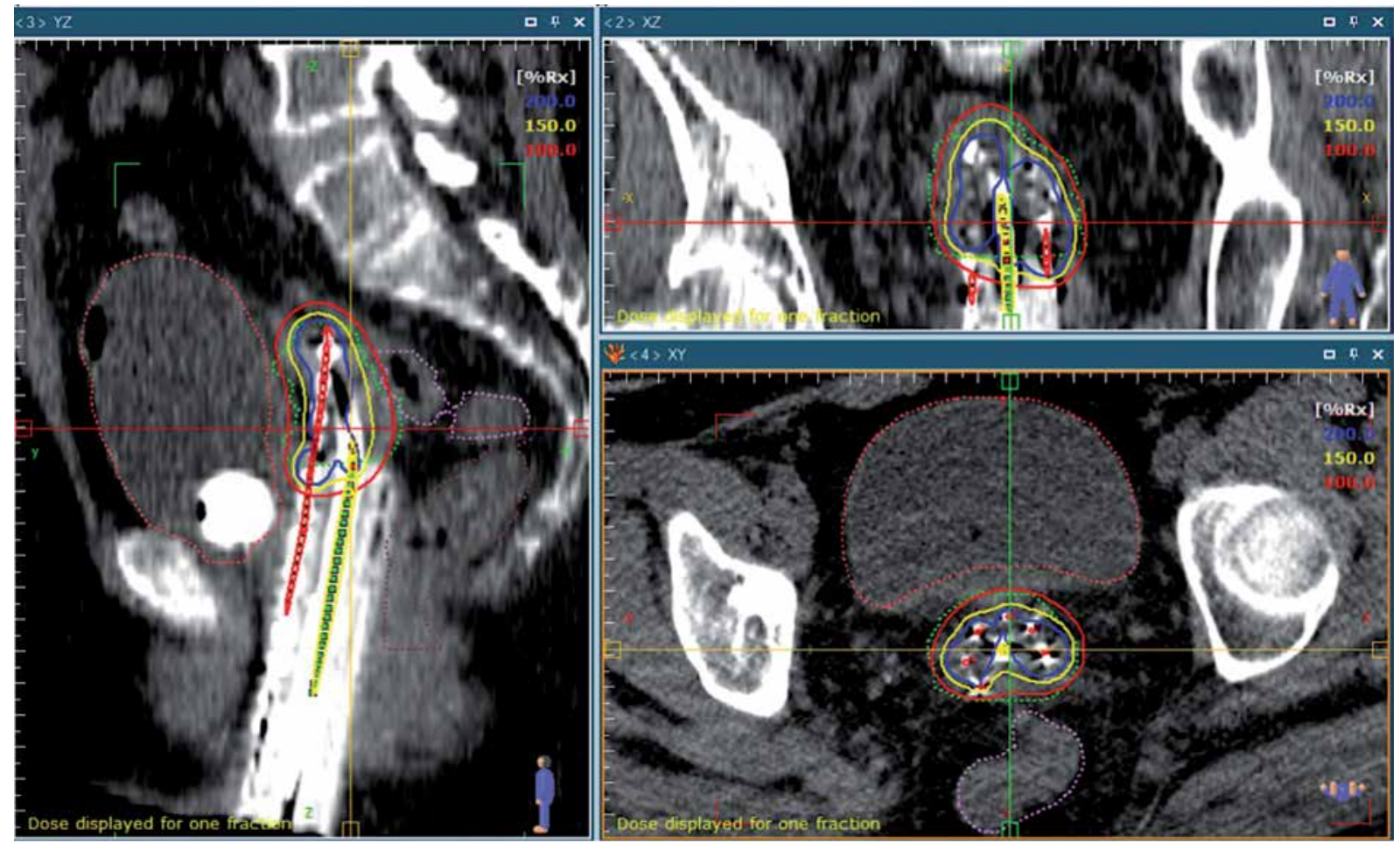

Fig. 6. A patient who treated with intrauterine, cylinder, and needles (TCN). A) Planning based on just activating intrauterine. B) Planning based on activating intrauterine and peripheral needles. Bladder - light red, rectum - brown, sigmoid - pink, and high-risk CTV (HR-CTV) - green

es of film measurements for 24 out of $192(12.5 \%)$ control points were greater than those of Monte Carlo calculations. Measurement dose, Monte Carlo results, and calculated dose from TPS for various control points are summarized in Table 3. P-value for dose difference between film measurement and TPS, Monte Carlo and TPS, and film measurement and Monte Carlo were 0.7, 0.66, 0.95, respectively. Therefore, no statistical difference between each group was observed.

Target coverage for the case report using just intrauterine probe was less than that used in TCN, due to an increase of sigmoid dose. The largest width of HR-CTV 
Table 2. Comparison results between film measurement, planning, and Monte Carlo

\begin{tabular}{lccc} 
& Mean \pm SD (Gy) & Mean \pm SD (\%) & $P$-value \\
\hline Film measurement vs. calculated doses in the TPS & $0.0860 \pm 0.0393$ & $4.01 \pm 1.21$ & 0.70 \\
\hline Film measurement vs. Monte Carlo & $0.0136 \pm 0.0200$ & $0.60 \pm 0.69$ & 0.95 \\
\hline Calculated doses in TPS vs. Monte Carlo & $0.0996 \pm 0.0471$ & $4.58 \pm 1.05$ & 0.66
\end{tabular}

was $50 \mathrm{~mm}$ for this patient, and the insertion of needles improved tumor coverage. It increased from $80 \%$ to $100 \%$ of prescription dose of tumor, while decreased sigmoid dose to $2 \mathrm{~cm}^{3}$ from $71 \%$ to $62 \%$ of prescription dose. Dose reduction for bladder and rectum were not significant (below 1\%) compared to sigmoid.

\section{Discussion}

In this study, we designed and manufactured a multi-channel cylinder applicator (MCCA) with 3D printing technology, and then evaluated its dosimetric characteristics with film measurement and Monte Carlo simulation. We determined control points around the applicator, and the dose in all control points from the TPS were compared to the results of film measurement and Monte Carlo simulation by using statistical analysis. There were no significant differences between the dose from TPS and film measurement and Monte Carlo algorithm for all control points.

It is possible to improve coverage of tumors located at one side of vagina by choosing suitable needles in the MCCA. Kim et al. [9] compared multi-channel and single-channel cylinder applicators for vagina cancers. They found that there were significant differences between multi-channel and single-channel applicators for the dose to rectum and tumor coverage. When tumors were located in the vagina cuff, dosimetry results were the same for both applicators. The multi-channel applicator was superior for the vagina wall tumors with width less than $7 \mathrm{~mm}$. For thicker tumors, the authors recommended interstitial brachytherapy to decrease vagina surface dose. For such patients, with 3D printing technology, it is possible to acquire CT scan before implantation (for pre-planning) and design a patient-specific multi-channel applicator with oblique needles, based on tumor shape and location or any arbitrary direction for better coverage of thicker tumors. For cervical cancer patients with parametria involvement, the insertion of needles for better coverage is preferable. It is easier to insert needles to the parametria region with TCN against to insertion of needles from perineal region, which needs an expert physician.

Currently, there are different 3D printing technologies, including fused deposition modeling, selective laser sintering, stereolithography, binder jetting, multi jet fusion, and microstereolithography, which allow users to print with different accuracy (up to less than $10 \mu \mathrm{m}$ ). At this time, there are limitations in the choice of material based on choosing a 3D printing technique. In order to select a material, biocompatibility, serializable and homogenic printing product, and water equivalency (specially for TPS with TG-43 algorithm) for brachytherapy need to be considered. Most of the studies used PA, PLA, ABS, and PC-ISO as a material with fused deposition modeling (known as FDM) technique $[19,21,29,39,40]$. Resin is yet another material with different sub-types, including rigid resin, tough resin, elastic resin, flexible resin, and high-temp resin for different purposes, with almost the same density. However, till date, no study investigated brachytherapy application using high-temp resin. High temperature resin has a heat deflection of $238^{\circ} \mathrm{C}$ and $0.45 \mathrm{MPa}$, which is the highest temperature resistance 3D printing material on the market, which can be sterilized with an autoclave [41]. Cunha et al. [19] used PCISO material to create a cylinder, resulting with better HU close to the water compared to our results (10 against $252)$, but the mechanical accuracy of our printed applicator was better comparing to their results, which was due to the differences between 3D printing technique accuracy. HU and density of printed material with FDM technique depend on choosing infill percentage. Ricotti et al. [39] assessed the dosimetric properties at different infill percentage. They found that $\gamma$ passing rate between 3D-printed and commercial applicator was depending on infill percentage, and even $\mathrm{HU}$ and density might be close to water by choosing infill percentage. Also, they used FDM technique to create an applicator, and differences between designed and 3D-printed geometry was higher than our results. The default of infill percentage of SLA was $100 \%$, and could not be controlled to acquire different HU and density. Moreover, apart from temperature resistance, biocompatibility and water equivalent property of 3D printing materials, tensile strength, young's modulus, elongation, and flexural strength are important and shown in the resistance of material to break under tension, stretch under tension, breaking when stretched, and breaking when bent. Resin has higher values compared to materials that are commonly used for FDM technique [42].

There were some limitations in our study, including dose calculation of TPS based on TG-43 formalism, which cannot consider heterogeneity effect (causing differences between TPS and Monte Carlo results), biocompatibility assessment of high-temp resin materials, dimension of the phantom that did not provide full scatter condition, uncertainty of Gafchromic film dosimetry, dose comparison with limited number of control points, lack of clinical study to compare target coverage and organ at risks doses with MCCA and single-channel approach statistically, and assessment of MCCA with simple design. We manufactured MCCA with $25 \mathrm{~mm}$ as a diameter in this study to assess dosimetry and radiological properties of resin material, but it is possible to print any arbitrary diameter and length for individual patient. In this study, we focused on 
Table 3. Measurement dose, Monte Carlo results, and calculated dose from TPS for various control points

$\begin{array}{lllll}\begin{array}{l}\text { Label } \\ \text { of control } \\ \text { points }\end{array} & \text { TPS doses (Gy) } & \begin{array}{c}\text { Monte Carlo } \\ \text { results (Gy) }\end{array} & \begin{array}{c}\text { Measurement } \\ \text { dose (Gy) }\end{array} & \begin{array}{c}\text { Difference be- } \\ \text { tween TPS and } \\ \text { Monte Carlo (\%) }\end{array}\end{array}$

$\begin{array}{cc}\text { Difference be- } & \text { Difference } \\ \text { tween TPS and } & \text { between } \\ \text { measurement } & \text { measurement and } \\ (\%) & \text { Monte Carlo (\%) }\end{array}$

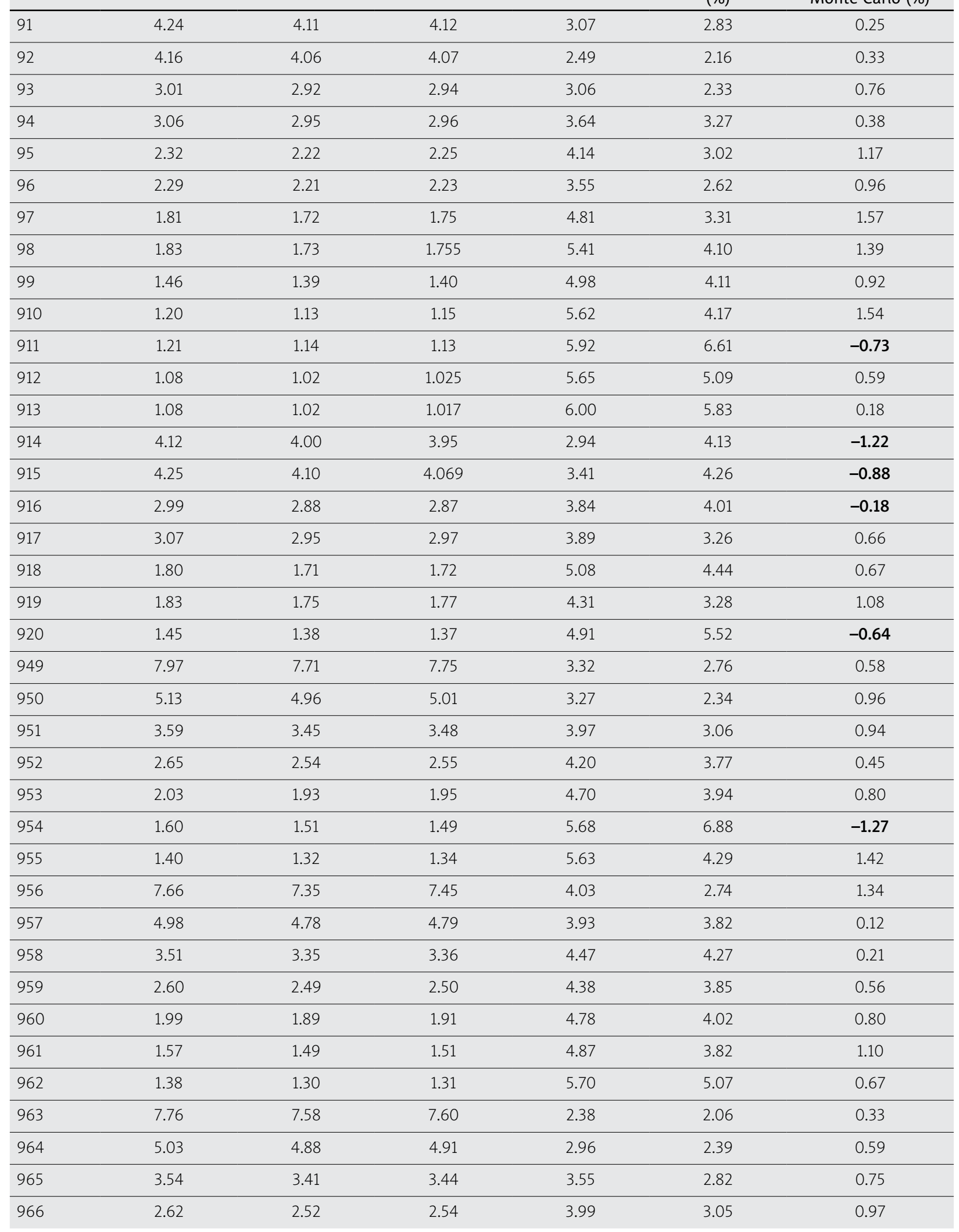


mechanical and dosimetric properties of high-temp resin and 3D printing workflow of patient-specific applicators.

\section{Conclusions}

In this study we used 3D printing technology to manufacture patient-specific MCCA. Dosimetric results and mechanical accuracy show that high-temp resin with SLA 3D printing technique can be used for creating patient-specific MCCA in brachytherapy.

\section{Disclosure}

The authors report no conflict of interest.

\section{References}

1. Creutzberg CL, van Putten WL, Koper PC et al. Surgery and postoperative radiotherapy versus surgery alone for patients with stage-1 endometrial carcinoma: multicentre randomised trial. Lancet 2000; 355: 1404-1411.

2. Keys HM, Roberts JA, Brunetto VL et al. A phase III trial of surgery with or without adjunctive external pelvic radiation therapy in intermediate risk endometrial adenocarcinoma: a Gynecologic Oncology Group study. Gynecol Oncol 2004 92: 744-751.

3. Kharouta MZ, Pham N, Nieto K et al. Comparison of dosimetric and clinical outcomes between short- and long-channel cylinder applicators for vaginal brachytherapy in intermediate- and high-risk endometrial cancer. Brachytherapy 2018; 17: 673-679.

4. Nout RA, Smit VT, Putter H et al. Vaginal brachytherapy versus pelvic external beam radiotherapy for patients with endometrial cancer of high-intermediate risk (PORTEC-2): an open-label, non-inferiority, randomised trial. Lancet 2010; 375: 816-823.

5. Sorbe B, Horvath G, Andersson $\mathrm{H}$ et al. External pelvic and vaginal irradiation versus vaginal irradiation alone as postoperative therapy in medium-risk endometrial carcinoma--a prospective randomized study. Int J Radiat Oncol Biol Phys 2012; 82: 1249-1255.

6. de Boer SM, Nout RA, Jurgenliemk-Schulz IM et al. Longterm impact of endometrial cancer diagnosis and treatment on health-related quality of life and cancer survivorship: results from the randomized PORTEC-2 trial. Int I Radiat Oncol Biol Phys 2015; 93: 797-809.

7. Kunneman M, Pieterse AH, Stiggelbout AM et al. Treatment preferences and involvement in treatment decision making of patients with endometrial cancer and clinicians. $\mathrm{Br} \mathrm{J}$ Cancer 2014; 111: 674-679.

8. Glaser S, Kim H, Beriwal S. Multi-channel vaginal cylinder brachytherapy: impact of tumor size and location on dose to organs at risk. Brachytherapy 2015; 14: 913-918.

9. Kim H, Rajagopalan MS, Houser C et al. Dosimetric comparison of multichannel with one single-channel vaginal cylinder for vaginal cancer treatments with high-dose-rate brachytherapy. Brachytherapy 2014; 13: 263-267.

10. Small W, Jr., Beriwal S, Demanes DJ et al. American Brachytherapy Society consensus guidelines for adjuvant vaginal cuff brachytherapy after hysterectomy. Brachytherapy 2012; 11: 58-67.

11. Qian JM, Stahl JM, Young MR et al. Impact of vaginal cylinder diameter on outcomes following brachytherapy for early stage endometrial cancer. J Gynecol Oncol 2017; 28: e84.

12. Zhang H, Gopalakrishnan M, Lee P et al. Dosimetric impact of cylinder size in high-dose rate vaginal cuff brachytherapy
(VCBT) for primary endometrial cancer. J Appl Clin Med Phys 2016; 17: 262-272.

13. Onal C, Guler O, Dolek Y. The impact of air pockets around the vaginal cylinder on vaginal vault brachytherapy. $\mathrm{Br} J \mathrm{Ra}$ diol 2015; 88: 20140694.

14. Richardson S, Palaniswaamy G, Grigsby PW. Dosimetric effects of air pockets around high-dose rate brachytherapy vaginal cylinders. Int J Radiat Oncol Biol Phys 2010; 78: 276-279.

15. Sethi R, Cunha A, Mellis K et al. Clinical applications of custom-made vaginal cylinders constructed using three-dimensional printing technology. J Contemp Brachytherapy 2016; 8: 208-214.

16. Lindegaard JC, Madsen ML, Traberg A et al. Individualised 3D printed vaginal template for MRI guided brachytherapy in locally advanced cervical cancer. Radiother Oncol 2016; 118: 173-175.

17. Zhao Z, Tang $X$, Mao $Z$ et al. The design of an individualized cylindrical vaginal applicator with oblique guide holes using 3D modeling and printing technologies. J Contemp Brachytherapy 2019; 11: 479-487.

18. Lee JH, Kim HN, Lim HS et al. Three-dimensional-printed vaginal applicators for electronic brachytherapy of endometrial cancers. Med Phys 2019; 46: 448-455.

19. Cunha JAM, Mellis K, Sethi R et al. Evaluation of PC-ISO for customized, 3D printed, gynecologic HDR brachytherapy applicators. J Appl Clin Med Phys 2015; 16: 246-253.

20. Sekii S, Tsujino K, Kosaka K et al. Inversely designed, 3D-printed personalized template-guided interstitial brachytherapy for vaginal tumors. J Contemp Brachytherapy 2018; 10: 470-477.

21. Sethi R, Cunha A, Mellis K et al. Clinical applications of custom-made vaginal cylinders constructed using three-dimensional printing technology. J Contemp Brachytherapy 2016; 8: 208-214.

22. Wiebe E, Easton $\mathrm{H}$, Thomas $\mathrm{G}$ et al. Customized vaginal vault brachytherapy with computed tomography imaging-derived applicator prototyping. Brachytherapy 2015; 14: 380-384.

23. Nilsson S, Moutrie Z, Cheuk R et al. A unique approach to high-dose-rate vaginal mold brachytherapy of gynecologic malignancies. Brachytherapy 2015; 14: 267-272.

24. Park SY, Kang S, Park JM et al. Development and dosimetric assessment of a patient-specific elastic skin applicator for highdose-rate brachytherapy. Brachytherapy 2019; 18: 224-232.

25. Lancellotta V, Pagano S, Tagliaferri L et al. Individual 3-dimensional printed mold for treating hard palate carcinoma with brachytherapy: A clinical report. J Prosthet Dent 2019; 121: 690-693

26. Kadoya N, Abe K, Nemoto H et al. Evaluation of a 3D-printed heterogeneous anthropomorphic head and neck phantom for patient-specific quality assurance in intensity-modulated radiation therapy. Radiol Phys Technol 2019; 12: 351-356.

27. Huang W, Lu J, Chen KM et al. Preliminary application of 3D-printed coplanar template for iodine-125 seed implantation therapy in patients with advanced pancreatic cancer. World J Gastroenterol 2018; 24: 5280-5287.

28. Huang MW, Zhang JG, Zheng L et al. Accuracy evaluation of a 3D-printed individual template for needle guidance in head and neck brachytherapy. J Radiat Res 2016; 57: 662-667.

29. Harris BD, Nilsson S, Poole CM. A feasibility study for using ABS plastic and a low-cost 3D printer for patient-specific brachytherapy mould design. Australas Phys Eng Sci Med 2015; 38: 399-412.

30. Aristei C, Lancellotta V, Piergentini M et al. Individualized 3D-printed templates for high-dose-rate interstitial multicathether brachytherapy in patients with breast cancer. Brachytherapy 2019; 18: 57-62. 
31. Kalaskar DM. 3D printing in medicine. Woodhead Publishing 2017.

32. Rybicki FJ, Grant GT. 3D Printing in Medicine. Springer International Publishing, Cham 2017.

33. Basic mechanics of stereolithography (SLA) 3D printing. 29.01.2020; Available from: https://formlabs.com/blog/ultimate-guide-to-stereolithography-sla-3d-printing/.

34. Sears NA, Seshadri DR, Dhavalikar PS et al. A review of three-dimensional printing in tissue engineering. Tissue Eng Part B Rev 2016; 22: 298-310.

35. Cho DW, Kang HW. Microstereolithography-based computer-aided manufacturing for tissue engineering, in computer-aided tissue engineering. Methods Mol Biol 2012; 868: 341-356.

36. Sorriaux J, Kacperek A, Rossomme S et al. Evaluation of Gafchromic ${ }^{\circledR}$ EBT3 films characteristics in therapy photon, electron and proton beams. Phys Med 2013; 29: 599-606.

37. Rueden CT, Schindelin J, Hiner MC et al. ImageJ2: ImageJ for the next generation of scientific image data. BMC Bioinformatics 2017; 18: 529

38. Devic S, Seuntjens J, Sham E et al. Precise radiochromic film dosimetry using a flat-bed document scanner. Med Phys 2005; 32 (7 Part 1): 2245-2253.

39. Ricotti R, Vavassori A, Bazani A et al. 3D-printed applicators for high dose rate brachytherapy: Dosimetric assessment at different infill percentage. Phys Med 2016; 32: 1698-1706.

40. Oare C, Wilke C, Ehler E et al. Dose calibration of Gafchromic EBT3 film for Ir-192 brachytherapy source using 3D-printed PLA and ABS plastics. 3D Print Med 2019; 5: 3.

41. High Temp Resin for High Thermal Stability. 29.01.2020; Available from: https://formlabs.com/materials/engineering/\#high-temp-resin.

42. MostCommonMechanicalandThermalProperties.29.01.2020; Available from: https:/ / formlabs.com/blog/how-to-choosethe-right-3D-printing-material/. 